\title{
Metabolic disturbances of non-alcoholic fatty liver resemble the alterations typical for type 2 diabetes
}

Citation for published version (APA):

Brouwers, B., Schrauwen-Hinderling, V. B., Jelenik, T., Gemmink, A., Havekes, B., Bruls, Y., Dahlmans, D., Roden, M., Hesselink, M. K. C., \& Schrauwen, P. (2017). Metabolic disturbances of non-alcoholic fatty liver resemble the alterations typical for type 2 diabetes. Clinical Science, 131(15), 1905-1917. https://doi.org/10.1042/CS20170261

Document status and date:

Published: 01/08/2017

DOI:

10.1042/CS20170261

Document Version:

Publisher's PDF, also known as Version of record

Document license:

Taverne

Please check the document version of this publication:

- A submitted manuscript is the version of the article upon submission and before peer-review. There can be important differences between the submitted version and the official published version of record.

People interested in the research are advised to contact the author for the final version of the publication, or visit the DOI to the publisher's website.

- The final author version and the galley proof are versions of the publication after peer review.

- The final published version features the final layout of the paper including the volume, issue and page numbers.

Link to publication

\footnotetext{
General rights rights.

- You may freely distribute the URL identifying the publication in the public portal. please follow below link for the End User Agreement:

www.umlib.nl/taverne-license

Take down policy

If you believe that this document breaches copyright please contact us at:

repository@maastrichtuniversity.nl

providing details and we will investigate your claim.
}

Copyright and moral rights for the publications made accessible in the public portal are retained by the authors and/or other copyright owners and it is a condition of accessing publications that users recognise and abide by the legal requirements associated with these

- Users may download and print one copy of any publication from the public portal for the purpose of private study or research.

- You may not further distribute the material or use it for any profit-making activity or commercial gain

If the publication is distributed under the terms of Article $25 \mathrm{fa}$ of the Dutch Copyright Act, indicated by the "Taverne" license above, 


\title{
Metabolic disturbances of non-alcoholic fatty liver resemble the alterations typical for type 2 diabetes
}

\author{
Bram Brouwers ${ }^{1,2}$, Vera B. Schrauwen-Hinderling ${ }^{1,3}$, Tomas Jelenik ${ }^{4,5}$, Anne Gemmink ${ }^{1,2}$, Bas Havekes ${ }^{1,6}$, \\ Yvonne Bruls ${ }^{1,3}$, Dennis DahImans ${ }^{1,2}$, Michael Roden ${ }^{4,5,7}$, Matthijs K.C. Hesselink ${ }^{1,2}$ and Patrick Schrauwen ${ }^{1,2}$ \\ ${ }^{1}$ NUTRIM School of Nutrition and Translational Research in Metabolism, Maastricht University Medical Center, Maastricht, The Netherlands; ${ }^{2}$ Department of Human Biology and \\ Human Movement Sciences, Maastricht University Medical Center, Maastricht, The Netherlands; ${ }^{3}$ Department of Radiology, Maastricht University Medical Center, Maastricht, The \\ Netherlands; ${ }^{4}$ Institute for Clinical Diabetology, German Diabetes Center, Leibniz Center for Diabetes Research at Heinrich-Heine University Düsseldorf, Düsseldorf, Germany; \\ ${ }^{5}$ German Center for Diabetes Research, München-Neuherberg, Düsseldorf, Germany;Department of Internal Medicine, Division of Endocrinology, Maastricht University Medical \\ Center, Maastricht, The Netherlands ${ }^{6} ;{ }^{7}$ Division of Endocrinology and Diabetology, Medical Faculty, Heinrich-Heine University Düsseldorf, Düsseldorf, Germany
}

Correspondence: Patrick Schrauwen (p.schrauwen@maastrichtuniversity.nl)

Received: 27 March 2017

Revised: 12 June 2017

Accepted: 15 June 2017

Accepted Manuscript Online: 15 June 2017

Version of Record published: 7 July 2017
Non-alcoholic fatty liver (NAFL) is an independent risk factor for the development of type 2 diabetes (T2DM). We examined metabolic perturbations in patients with NAFL, patients with T2DM, and control (CON) subjects with normal intrahepatic lipid (IHL) content.

A two-step (10 $\left.\mathrm{mU} / \mathrm{m}^{2} / \mathrm{min} ; 40 \mathrm{mU} / \mathrm{m}^{2} / \mathrm{min}\right)$ hyperinsulinemic-euglycemic clamp was performed in 11 NAFL, 13 T2DM, and 11 CON subjects, all matched for BMI, and aerobic fitness. IHL content was measured using proton magnetic resonance spectroscopy. Because of high IHL content variability in T2DM patients, this group was separated into a high IHL content group (IHL $\geqslant 5.0 \%$, T2DM+NAFL) and a normal IHL content group $(\mathrm{IHL}<5.0 \%$, T2DM-non-NAFL) for further analysis.

IHL content was increased in NAFL and T2DM+NAFL subjects $(P<0.050$ versus $C O N$ and T2DM-non-NAFL subjects). Adipose tissue insulin sensitivity index (Adipo- $I \mathrm{R}_{\mathrm{i}}$ ) was higher in NAFL $(P<0.050$ versus CON and T2DM-non-NAFL subjects) and in T2DM+NAFL subjects ( $P=0.055$ versus $C O N$ subjects, $P<0.050$ versus T2DM-non-NAFL subjects). Suppression of plasma-free fatty acids $(P=0.046)$ was lower in NAFL compared with CON subjects, with intermediate values for T2DM-non-NAFL, and T2DM+NAFL subjects. Suppression of endogenous glucose production (EGP) and insulin-stimulated glucose disposal $\left(\Delta R_{\mathrm{d}}\right)$ was comparable between NAFL, T2DM-non-NAFL, and T2DM+NAFL subjects (all $P>0.05$ ), and was lower in comparison with CON subjects (all $P<0.01$ ). Metabolic flexibility was lower in T2DM-non-NAFL subjects $(P=0.047)$ and NAFL subjects $(P=0.059)$ compared with CON subjects. Adipo- $I \mathrm{R}_{\mathrm{i}}(r=0.652, P<0.001)$, hepatic insulin resistance index $\left(H I \mathrm{R}_{\mathrm{i}}\right)(r=0.576$, $P=0.001)$, and $\Delta R_{\mathrm{d}}(r=-0.653, P<0.001)$ correlated with IHL content.

Individuals with NAFL suffer from metabolic perturbations to a similar degree as T2DM patients. NAFL is an important feature leading to severe insulin resistance and should be viewed as a serious health threat for the development of T2DM. ClinicalTrials.gov: NCT01317576 
considered to be an independent risk factor for the development of type 2 diabetes (T2DM) [2,5]. Importantly, the prevalence of prediabetes and diabetes in individuals with NAFL is as high as $85 \%$, compared with only $30 \%$ in individuals with normal IHL content [6].

Elevated levels of alanine amino transferase (ALT) in plasma-a biomarker for liver disease-are known to be associated with the metabolic syndrome $[7,8]$. Cross-sectional studies using proton magnetic resonance spectroscopy $\left({ }^{1} \mathrm{H}-\mathrm{MRS}\right)$ - the non-invasive gold standard to measure IHL content in humans-found that elevated IHL content directly correlates with progressive impairments of adipose tissue, hepatic, and skeletal muscle insulin sensitivity in lean, overweight and obese non-diabetic subjects, and in patients with T2DM [9-14]. Furthermore, patients with NAFL are characterized by lower adipose tissue, hepatic, and skeletal muscle insulin sensitivity compared with healthy individuals with normal IHL content [15-18]. However, the severity of insulin resistance and metabolic disturbances in individuals with elevated IHL content but without T2DM when compared with patients with T2DM is currently unknown.

Therefore, the purpose of the present study was to perform a very detailed metabolic characterization of patients with NAFL, and compare them with BMI-matched, non-insulin-dependent patients with T2DM, and individuals with normal IHL content (control subjects: CON). Based on the current scientific consensus, which considers NAFL a prediabetic state, and based on previous results obtained in other individuals with prediabetes [19,20], we hypothesized that metabolic disturbances, such as adipose tissue, hepatic, and skeletal muscle insulin resistance and impaired metabolic flexibility, would have intermediate values in NAFL subjects compared with insulin-resistant T2DM patients and insulin-sensitive CON subjects.

\section{Experimental Participants}

In the study, 11 sedentary, middle-aged (40-70 years), BMI-matched $(27-35 \mathrm{~kg} / \mathrm{m} 2)$, overweight/obese NAFL subjects; 13 T2DM patients; and 11 CON subjects were included. All subjects were male, with stable dietary habits. General exclusion criteria were unstable body weight, cardiac disease, impaired renal function, anemia (hemoglobin $<7.5 \mathrm{mmol} / \mathrm{l})$, use of $\beta$-blockers, use of anti-thrombotic medication, elevated blood pressure ( $>160 / 100 \mathrm{mmHg}$ ), claustrophobia, contra-indications for MRI, recent participation in a weight loss or vigorous exercise program, history of substantial alcohol use ( $>3$ units per day), history of drug abuse, and use of insulin therapy. NAFL subjects were selected who had a high IHL content (all $\geqslant 5.0 \%)$ as measured with ${ }^{1} \mathrm{H}$-MRS, and a fasting plasma glucose (FPG) concentration $<7.0 \mathrm{mmol} / \mathrm{l}$. CON subjects were selected who had a low IHL content (all $\leqslant 4.0 \%$ ) as measured with ${ }^{1} \mathrm{H}-\mathrm{MRS}$, in the absence of clinical signs of liver disease or liver dysfunction (defined as alanine aminotransferase $($ ALAT) $>2.5$ times the normal value) and had to be normoglycemic as per WHO criteria. A 120-min $75 \mathrm{~g}$ oral glucose tolerance test (OGTT) was performed in NAFL and CON subjects. T2DM patients were treated for their hyperglycemia with metformin $(n=5)$, sulfonyl urea $(n=1)$, metformin + sulfonylurea $(n=4)$, metformin + dipeptidyl peptidase-4 (DPP-4) inhibitors $(n=1)$ or metformin + sulfonylurea + DPP-4 inhibitors $(n=1)$ therapy for at least 6 months with a constant dose for at least 2 months or were on a dietary treatment $(n=1)$ for at least 6 months prior to the onset of the study, with FPG concentration $\geqslant 7.0 \mathrm{mmol} / \mathrm{l}$, and $<10.0 \mathrm{mmol} / \mathrm{l}$ at onset of the study. Participants were recruited via advertisements in local newspapers and gave written informed consent before participation. All data were collected at the Maastricht University Medical Center, Maastricht, The Netherlands. The local ethics committee approved the study, which was performed following the declaration of Helsinki principles.

\section{Body composition and maximal aerobic capacity $\left(\mathrm{VO}_{2 \max }\right)$}

Body composition was determined by dual X-ray absorptiometry (Hologic Discovery A, Waltham, MA, U.S.A.). Waist circumference was assessed at the end of exhalation, with the subjects standing erect, and relaxed with arms at their sides and their feet positioned close together. The waist circumference was measured to the nearest $0.1 \mathrm{~cm}$ in a horizontal plane around the abdomen at the level of the iliac crest. $V \mathrm{O}_{2 \max }$ and $W_{\max }$ were assessed during a graded cycling test with concurrent ECG until exhaustion, as previously described [21].

\section{${ }^{1} \mathrm{H}-\mathrm{MRS}$}

${ }^{1}$ H-MRS was used to quantify IHL content, as described previously [22], but on a 3T whole-body scanner (Achieva 3Tx; Philips Healthcare, Best, The Netherlands) using a five-element coil, and a repetition time $=4000 \mathrm{~ms}$, echo time $=32.5 \mathrm{~ms}$ and number of averages $=64$. To minimize motion artifacts, subjects were asked to breathe in to the rhythm of the measurement and to be at end of expiration during acquisition of spectra. To determine the intensity of the lipid peak, the water signal was suppressed using frequency-selective pre-pulses. The unsuppressed water resonance 
was used as an internal reference (number of averages $=32$ ). Spectra were fitted with a home-written script [23] in MATLAB R2014b (Mathworks, Natick, MA, U.S.A.). The $\mathrm{CH}_{2}$ resonance and the unsuppressed water resonance were corrected for T2 relaxation according to [24] and IHL content was calculated in weight/weight percentage, as described previously [25].

\section{Hyperinsulinemic-euglycemic clamp}

All participants underwent a two-step $\left(10 \mathrm{mU} / \mathrm{m}^{2} / \mathrm{min} ; 40 \mathrm{mU} / \mathrm{m}^{2} / \mathrm{min}\right)$ hyperinsulinemic-euglycemic clamp for $6 \mathrm{~h}$ [26], as described previously [27]. In short, after an overnight fast, participants received a primed continuous infusion of $\left[6,6-{ }^{2} \mathrm{H}_{2}\right]$-glucose $(0.04 \mathrm{mg} / \mathrm{kg} / \mathrm{min})$. After $180 \mathrm{~min}, 10 \mathrm{mU} / \mathrm{m}^{2} / \mathrm{min}$ insulin infusion was started for $4 \mathrm{~h}$. Thereafter, $40 \mathrm{mU} / \mathrm{m}^{2} / \mathrm{min}$ insulin infusion was started for $2 \mathrm{~h}$. During insulin infusion, plasma glucose concentrations were maintained by a concomitant infusion of glucose that was enriched with $\left[6,6-{ }^{2} \mathrm{H}_{2}\right]$-glucose (hot-GINF) to minimize changes in plasma tracer enrichment [28]. Glucose steady state was reached during the last 30 min of both insulin infusion rates. Blood sampling and indirect calorimetry using ventilated hood system (Omnical, IDEE, Maastricht, The Netherlands) was performed in every final $30 \mathrm{~min}$ of steady state. Isotopic enrichment of plasma glucose was determined by electric impact ionization gas chromatography mass spectroscopy as previously described [29].

\section{Calculations}

Whole-body insulin sensitivity was assessed as the mean glucose infusion rate during $40 \mathrm{mU} / \mathrm{m}^{2} / \mathrm{min}$ insulin infusion $(M$-value, $\mu \mathrm{mol} / \mathrm{min} / \mathrm{kgFFM})$, and as $M$-value per mean plasma insulin concentration (I, mU/l) during 40 $\mathrm{mU} / \mathrm{m}^{2} / \mathrm{min}$ insulin infusion (M/I ratio). Steele's single-pool, non-steady-state equations were used to calculate glucose rate of appearance $\left(R_{\mathrm{a}}\right)$, and glucose rate of disappearance $\left(R_{\mathrm{d}}\right)$ [30]. Volume of distribution was assumed to be $0.160 \mathrm{l} / \mathrm{kg}$ for glucose. Insulin-stimulated glucose disposal $\left(\Delta R_{\mathrm{d}}\right)$ was computed as the difference between $R_{\mathrm{d}}$ under insulin-stimulated conditions $\left(40 \mathrm{mU} / \mathrm{m}^{2} / \mathrm{min}\right)$ and $R_{\mathrm{d}}$ under basal conditions. Suppression of endogenous glucose production (EGP) was calculated as $R_{\mathrm{a}}$ - exogenous glucose infusion rate. The difference in respiratory quotient (RQ) under insulin-stimulated conditions $\left(40 \mathrm{mU} / \mathrm{m}^{2} / \mathrm{min}\right)$ and RQ under basal conditions was defined as metabolic flexibility $(\triangle \mathrm{RQ})$. Glucose and fat oxidation rates were calculated with the assumption that protein oxidation was negligible [31]. Non-oxidative glucose disposal (NOGD) was calculated as $R_{\mathrm{d}}\left(40 \mathrm{mU} / \mathrm{m}^{2} / \mathrm{min}\right)$ - glucose oxidation $\left(40 \mathrm{mU} / \mathrm{m}^{2} / \mathrm{min}\right)$. The hepatic insulin resistance index $\left(\mathrm{HIR}_{\mathrm{i}}\right)$ and adipose tissue insulin resistance index (Adipo- $\mathrm{IR}_{\mathrm{i}}$ ) were calculated as previously described [12]. $\mathrm{HIR}_{\mathrm{i}}$ was calculated as the product of basal EGP and basal plasma insulin concentration before the start of the hyperinsulinemic-euglycemic clamp. Adipo-IR $\mathrm{R}_{\mathrm{i}}$ was calculated as the product of basal plasma free fatty acids (FFA) and basal plasma insulin concentration before the start of the hyperinsulinemic-euglycemic clamp.

\section{Laboratory analysis}

Arterialized blood samples were collected and immediately centrifuged at high speed. Plasma was frozen in liquid nitrogen and stored at $-80^{\circ} \mathrm{C}$ until assayed. Plasma FFAs and plasma glucose were measured with enzymatic assays automated on a Cobas Fara/Mira (FFA: Wako Nefa C test kit; Wako Chemicals, Neuss, Germany) (plasma glucose: hexokinase method; La Roche, Basel, Switzerland). Fasting plasma triglycerides (TG) were measured colorimetrically (Roche, Vienna, Austria). Total cholesterol (Roche Diagnostics, Mannheim, Germany) and high-density lipoprotein (HDL) (Roche Diagnostics, Mannheim, Germany), after precipitation of apolipoprotein B-containing lipoproteins with phosphotungstic acid, and magnesium ions, were analyzed in serum enzymatically (GPO-Trinder; Sigma-Aldrich Corp., St. Louis, MO, U.S.A.). Low-density lipoprotein (LDL) was calculated according to the Friedewald equation [32], with no subjects having plasma TG levels above $4.5 \mathrm{mmol} / \mathrm{l}$. Plasma insulin concentrations on the day of the hyperinsulinemic-euglycemic clamp were quantified using an immunometric assay (Advia Centaur, Siemens Diagnostics). Blood parameters during the screening visit were analyzed routinely.

\section{Statistics}

All values are reported as the mean \pm SEM. Statistical significance was set at $P<0.05$. Normality was assessed by the Shapiro-Wilk test. Homogeneity of variance was assessed by Levene's test. Group comparisons were performed using one-way ANOVA with Tukey or Games-Howell post-hoc correction, or using Kruskal-Wallis H test with Dunn's (1964) procedure with Bonferroni correction for multiple comparison. For Pearson correlations, logarithmic transformation of IHL content, Glucose $120, \mathrm{HIR}_{\mathrm{i}}$, and Adipo- $\mathrm{IR}_{\mathrm{i}}$ was performed as the data were skewed. All statistic calculations were performed using IBM SPSS 21 (SPSS, Chicago, IL, U.S.A.). 
Table 1 Subject characteristics

\begin{tabular}{|c|c|c|c|c|c|}
\hline & CON & NAFL & T2DM-non-NAFL & T2DM+NAFL & $P$ value \\
\hline Age (years) & $57.6 \pm 2.5$ & $54.5 \pm 2.0$ & $63.0 \pm 1.3$ & $59.8 \pm 3.2$ & 0.095 \\
\hline $\mathrm{IHL}(\%)$ & $1.9 \pm 0.4^{\mathrm{A}}$ & $9.7 \pm 2.0^{\mathrm{B}}$ & $2.3 \pm 0.4^{\mathrm{A}}$ & $16.6 \pm 6.0^{\mathrm{B}}$ & $<0.001$ \\
\hline AST (U/l) & $22.1 \pm 1.2$ & $25.5 \pm 2.4$ & $24.0 \pm 1.9$ & $29.5 \pm 3.4$ & 0.176 \\
\hline ALT (U/I) & $30.0 \pm 3.6$ & $34.5 \pm 2.2$ & $32.1 \pm 3.5$ & $45.5 \pm 7.4$ & 0.078 \\
\hline$\gamma$-GT (U/I) & $34.3 \pm 4.7$ & $35.4 \pm 3.7$ & $39.0 \pm 5.8$ & $42.8 \pm 9.1$ & 0.726 \\
\hline Body weight (kg) & $93.8 \pm 3.6$ & $102.2 \pm 2.9$ & $90.4 \pm 2.7$ & $97.5 \pm 3.1$ & 0.079 \\
\hline Height (cm) & $178.0 \pm 5.9$ & $181.9 \pm 7.2$ & $177.7 \pm 1.8$ & $177.2 \pm 3.0$ & 0.330 \\
\hline $\mathrm{BMI}\left(\mathrm{kg} / \mathrm{m}^{2}\right)$ & $29.5 \pm 0.8$ & $30.9 \pm 0.8$ & $28.8 \pm 0.8$ & $31.0 \pm 0.6$ & 0.187 \\
\hline Fat mass $(\mathrm{kg})$ & $27.5 \pm 1.9$ & $30.7 \pm 1.2$ & $25.4 \pm 1.6$ & $29.0 \pm 1.0$ & 0.134 \\
\hline Fat free mass $(\mathrm{kg})$ & $64.2 \pm 1.9$ & $69.0 \pm 2.0$ & $63.8 \pm 1.3$ & $66.3 \pm 2.3$ & 0.200 \\
\hline Fat percentage (\%) & $28.8 \pm 1.0$ & $30.0 \pm 0.7$ & $27.5 \pm 1.2$ & $29.7 \pm 0.7$ & 0.326 \\
\hline Waist circumference (cm) & $106.2 \pm 1.9$ & $109.1 \pm 2.0$ & $105.2 \pm 1.8$ & $110.3 \pm 1.2$ & 0.332 \\
\hline Fasting glucose (mmol/l) & $5.1 \pm 0.2^{\mathrm{A}}$ & $5.5 \pm 0.1^{\mathrm{A}}$ & $7.5 \pm 0.4^{\mathrm{B}}$ & $7.4 \pm 0.6^{\mathrm{B}}$ & $<0.001$ \\
\hline Glucose $120(\mathrm{mmol} / \mathrm{l})$ & $4.8 \pm 0.3^{\mathrm{A}}$ & $6.7 \pm 0.7^{\mathrm{B}}$ & $\overline{N D}$ & $\overline{N D}$ & 0.021 \\
\hline Fasting insulin (mU/l) & $9.2 \pm 0.7$ & $15.2 \pm 1.9$ & $11.4 \pm 3.1$ & $19.1 \pm 4.1$ & 0.020 \\
\hline Triglycerides (mmol/l) & $1.3 \pm 0.2$ & $1.7 \pm 0.2$ & $1.3 \pm 0.2$ & $1.9 \pm 0.3$ & 0.136 \\
\hline Total cholesterol & $4.7 \pm 0.3$ & $4.9 \pm 0.3$ & $4.0 \pm 0.3$ & $3.9 \pm 0.4$ & 0.144 \\
\hline LDL & $3.2 \pm 0.3$ & $3.4 \pm 0.3$ & $2.9 \pm 0.3$ & $2.4 \pm 0.3$ & 0.184 \\
\hline $\mathrm{HDL}$ & $1.2 \pm 0.1$ & $1.1 \pm 0.1$ & $1.0 \pm 0.1$ & $1.1 \pm 0.1$ & 0.283 \\
\hline$V O_{2 \max }(\mathrm{ml} / \mathrm{min})$ & $2574.3 \pm 128.1$ & $2658.6 \pm 151.5$ & $2407.0 \pm 126.1$ & $2361.1 \pm 115.9$ & 0.443 \\
\hline$V O_{2 \max }(\mathrm{ml} / \mathrm{min} / \mathrm{kg})$ & $27.6 \pm 1.4$ & $26.1 \pm 1.4$ & $26.7 \pm 1.5$ & $24.2 \pm 1.0$ & 0.472 \\
\hline$W_{\max }(\mathrm{W} / \mathrm{kg})$ & $2.1 \pm 0.1$ & $1.9 \pm 0.1$ & $1.9 \pm 0.1$ & $1.8 \pm 0.1$ & 0.401 \\
\hline
\end{tabular}

Abbreviations: ALT: alanine aminotransferase; AST: aspartate aminotransferase; BMI: body mass index; HDL: high-density lipoprotein; IHL: intrahepatic lipid; LDL: low-density lipoprotein; VAT: visceral adipose tissue; $V_{2} O_{2 m a x}$ : maximal oxidative capacity; $W_{\max }$ : maximal output; $\gamma$-GT: gamma-glutamyl transferase; ND, not determined. Different letters indicate significant differences between groups $(P<0.05)$.

\section{Results \\ Subject characteristics}

Subject characteristics are summarized in Table 1. Because T2DM subjects had a wide spread in IHL content, we decided to analyze T2DM subjects with high IHL content (IHL $\geqslant 5.0 \%$, T2DM+NAFL, $n=6$ ), and with normal IHL content (IHL $<5.0 \%$, T2DM-non-NAFL subjects, $n=7)$ separately.

Age $(P=0.095)$, BMI $(P=0.187)$, fat mass $(P=0.134)$, fat-free mass $(P=0.200)$, fat percentage $(P=0.326)$, and waist circumference $(P=0.332)$ were not different among groups. By design, screening FPG was significantly different among groups $(P<0.001)$. NAFL and CON subjects had similar screening FPG levels $(P=0.792)$ in the normoglycemic range. Compared with NAFL and CON subjects, T2DM-non-NAFL, and T2DM+NAFL subjects had increased screening FPG levels (all $P<0.05$ ). Screening FPG levels in T2DM-non-NAFL and T2DM+NAFL subjects were comparable $(P=0.992)$. Screening fasting insulin levels were different among groups $(P=0.020)$, with a trend for higher fasting insulin levels in NAFL $(P=0.088)$, and T2DM+NAFL subjects $(P=0.060)$ compared with CON subjects. Plasma glucose levels upon OGTT (glucose 120) were higher in NAFL than in CON subjects $(P=0.021)$. For T2DM-non-NAFL and T2DM+NAFL, no OGTT was performed. Fasting plasma TG levels $(P=0.136)$, total cholesterol $(P=0.144)$, LDL $(P=0.184)$, and $\operatorname{HDL}(P=0.283)$ were not different among groups. Aerobic fitness was comparable among groups, with no differences in absolute $V_{2 \max }(P=0.443), V \mathrm{O}_{2 \max }$ corrected for body weight $(P=0.472)$, and $W_{\max }(P=0.401)$.

\section{IHL content and liver enzymes}

By design, IHL content was different among groups $(P<0.001)$ (Figure $1 \mathrm{~A}$, Table 1). IHL content was comparable between NAFL and T2DM+NAFL subjects $(P=0.828)$ and between CON and T2DM-non-NAFL subjects $(P=0.887)$ (Figure 1A, Table 1). Glucose levels upon OGTT (glucose 120), which was only performed in CON and NAFL subjects, correlated with IHL content $(r=0.638, P=0.002)$.

Plasma levels of aspartate aminotransferase (AST) $(P=0.176)$, alanine aminotransferase (ALT) $(P=0.078)$, and gamma-glutamyl transferase $(\gamma$-GT $)(P=0.726)$ - markers of hepatocellular injury - were not different among groups (Figure 1B). ALT tended to be somewhat higher in T2DM+NAFL subjects due to a statistical but not clinical outlier 
A

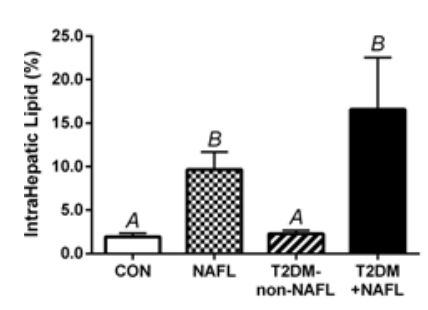

C

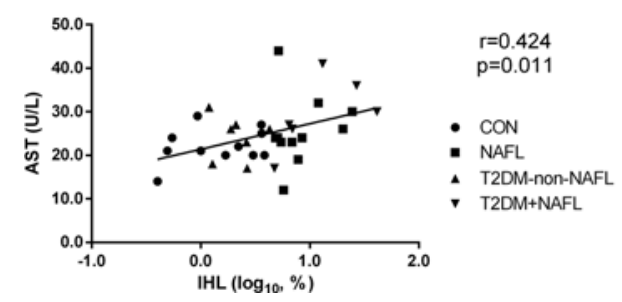

B

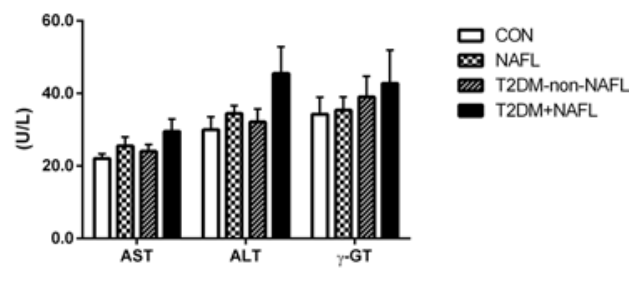

D

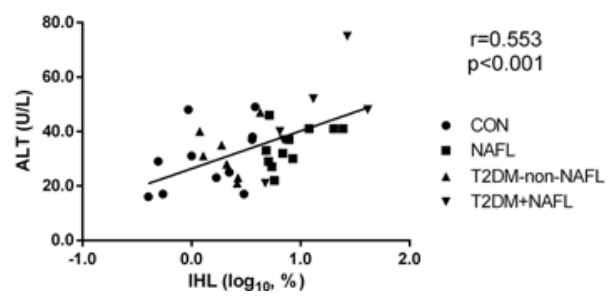

Figure 1. Intrahepatic lipid content and liver enzymes

(A) Intrahepatic lipid (IHL) content; (B) serum aspartate aminotransferase (AST), alanine aminotransferase (ALT), and gamma-glutamyl transferase ( $\gamma$-GT) levels; (C) relation between serum AST levels and IHL content $(n=35)$; (D) relation between serum ALT levels and IHL content $(n=35)$. Results are mean \pm SEM. Different letters indicate significant differences between groups $(P<0.05)$.

that remained included in the analysis. Despite no significant group differences, AST ( $r=0.424, P=0.011$, Figure 1C), and ALT $(r=0.553, P<0.001$, Figure 1D) both showed a positive correlation with IHL content in the whole study population.

\section{Circulating metabolites during hyperinsulinemic-euglycemic clamp}

Plasma insulin concentrations during both the $10 \mathrm{mU} / \mathrm{m}^{2} / \mathrm{min}$ and $40 \mathrm{mU} / \mathrm{m}^{2} / \mathrm{min}$ steady state period of the clamp were comparable among groups (Table 2). Plasma glucose values during $10 \mathrm{mU} / \mathrm{m}^{2} / \mathrm{min}$ insulin infusion were different among groups $(P=0.019$, Table 2$)$. T2DM+NAFL subjects had higher plasma glucose values during 10 $\mathrm{mU} / \mathrm{m}^{2} / \mathrm{min}$ insulin infusion than CON subjects $(P=0.026)$, with NAFL and T2DM-non-NAFL subjects having intermediate values (Table 2). Plasma glucose values during $40 \mathrm{mU} / \mathrm{m}^{2} / \mathrm{min}$ insulin infusion $(P=0.143)$ and fasting plasma FFA levels were not significantly different among groups $(P=0.574$, Table 2$)$.

\section{Insulin sensitivity}

\section{Whole-body insulin sensitivity}

Whole-body insulin sensitivity was assessed as $M$-value and $\mathrm{M} / \mathrm{I}$ ratio during $40 \mathrm{mU} / \mathrm{m}^{2} / \mathrm{min}$ insulin infusion. $M$-value was different among groups $(P<0.001$, Table 2$) . M$-value was comparable between NAFL and T2DM-non-NAFL subjects $(P=0.827)$; between NAFL and T2DM+NAFL subjects $(P=0.553)$; and between T2DM-non-NAFL and T2DM+NAFL subjects $(P=0.466)$ (Table 2$)$. NAFL subjects $(P<0.001)$, T2DM-non-NAFL subjects $(P<0.001)$ and T2DM+NAFL subjects $(P<0.001)$ were all characterized by a significantly lower $M$-value compared with CON subjects (Table 2). The M/I ratio was also different among groups $(P=0.004$, Table 2$)$. M/I ratio was comparable between NAFL and T2DM-non-NAFL subjects $(P=0.314)$; NAFL and T2DM+NAFL subjects $(P=0.372)$; T2DM-non-NAFL and T2DM-NAFL subjects $(P=0.100)$ and T2DM-non-NAFL and CON subjects $(P=0.105)$ (Table 2$)$. NAFL $(P=0.003)$ and T2DM+NAFL subjects $(P=0.001)$ were characterized by a significantly lower $\mathrm{M} / \mathrm{I}$ ratio compared with CON subjects (Table 2).

\section{Adipose tissue insulin sensitivity}

Adipose tissue insulin resistance during basal conditions was determined by using the validated Adipo- $\mathrm{IR}_{\mathrm{i}}[12,33]$. Adipo- $\mathrm{IR}_{\mathrm{i}}$ was different among groups $(P=0.006$, Figure $2 \mathrm{~A})$. Adipo- $\mathrm{IR}_{\mathrm{i}}$ in NAFL was comparable with Adipo-IR in T2DM+NAFL subjects $(P=0.947$, Figure $2 A)$. Adipo- $\mathrm{IR}_{\mathrm{i}}$ in T2DM-non-NAFL was comparable with Adipo-IR $\mathrm{I}_{\mathrm{i}}$ in 
Table 2 Substrate kinetics during hyperinsulinemic-euglycemic clamp

\begin{tabular}{|c|c|c|c|c|c|}
\hline & CON & NAFL & T2DM-non-NAFL & T2DM+NAFL & $P$ value \\
\hline \multicolumn{6}{|l|}{ Plasma insulin (mU/l) } \\
\hline Basal & $8.6 \pm 1.0^{\mathrm{A}}$ & $17.0 \pm 2.2^{B}$ & $6.4 \pm 0.8^{\mathrm{A}}$ & $18.9 \pm 4.7^{\mathrm{B}}$ & 0.002 \\
\hline $10 \mathrm{mU}$ & $29.1 \pm 2.5$ & $34.1 \pm 2.2$ & $30.3 \pm 8.4$ & $37.0 \pm 3.5$ & 0.335 \\
\hline $40 \mathrm{mU}$ & $127.5 \pm 10.2$ & $132.7 \pm 10.5$ & $105.8 \pm 11.1$ & $147.8 \pm 8.7$ & 0.154 \\
\hline \multicolumn{6}{|l|}{ Plasma glucose (mmol/l) } \\
\hline Basal & $5.4 \pm 0.2^{\mathrm{A}}$ & $5.6 \pm 0.2^{A}$ & $9.0 \pm 0.8^{\mathrm{B}}$ & $8.3 \pm 0.9^{\mathrm{B}}$ & $<0.001$ \\
\hline $10 \mathrm{mU}$ & $5.1 \pm 0.2^{\mathrm{A}}$ & $5.2 \pm 0.1^{\mathrm{AB}}$ & $5.6 \pm 0.2^{\mathrm{AB}}$ & $5.9 \pm 0.4^{\mathrm{B}}$ & 0.019 \\
\hline $40 \mathrm{mU}$ & $5.2 \pm 0.1$ & $5.0 \pm 0.2$ & $4.8 \pm 0.2$ & $5.3 \pm 0.1$ & 0.143 \\
\hline$M$-value $(\mu \mathrm{mol} / \mathrm{min} / \mathrm{kgFFM})$ & $47.2 \pm 2.6^{A}$ & $27.0 \pm 3.6^{B}$ & $28.0 \pm 2.5^{\mathrm{B}}$ & $24.2 \pm 2.9^{B}$ & $<0.001$ \\
\hline $\begin{array}{l}\mathrm{M} / \mathrm{I} \text { ratio } \\
(\mu \mathrm{mol} / \mathrm{min} / \mathrm{kgFFM}) /(\mathrm{mU} / \mathrm{l})\end{array}$ & $0.40 \pm 0.04^{\mathrm{A}}$ & $0.23 \pm 0.04^{\mathrm{B}}$ & $0.29 \pm 0.06^{\mathrm{AB}}$ & $0.17 \pm 0.03^{\mathrm{B}}$ & 0.004 \\
\hline \multicolumn{6}{|l|}{$\mathrm{EGP}(\mu \mathrm{mol} / \mathrm{min} / \mathrm{kgFFM})$} \\
\hline Basal & $11.6 \pm 0.4$ & $11.1 \pm 0.4$ & $13.4 \pm 1.1$ & $12.9 \pm 0.6$ & 0.123 \\
\hline $10 \mathrm{mU}$ & $4.3 \pm 0.5^{\mathrm{A}}$ & $7.9 \pm 1.2^{\mathrm{B}}$ & $9.2 \pm 0.9^{\mathrm{B}}$ & $9.2 \pm 0.8^{\mathrm{B}}$ & $<0.001$ \\
\hline$\delta \%$ & $-62.9 \pm 4.0^{\mathrm{A}}$ & $-30.6 \pm 8.2^{\mathrm{B}}$ & $-30.6 \pm 6.2^{\mathrm{B}}$ & $-27.9 \pm 7.6^{\mathrm{B}}$ & $<0.001$ \\
\hline $40 \mathrm{mU}$ & $-2.0 \pm 0.5^{\mathrm{A}}$ & $0.17 \pm 0.6^{\mathrm{AB}}$ & $1.8 \pm 1.5^{\mathrm{B}}$ & $0.5 \pm 0.8^{\mathrm{AB}}$ & 0.018 \\
\hline$\delta \%$ & $--117.5 \pm 4.4^{\mathrm{A}}$ & $-98.7 \pm 5.9^{\mathrm{AB}}$ & $-86.2 \pm 13.3^{\mathrm{B}}$ & $-96.5 \pm 6.5^{\mathrm{B}}$ & 0.030 \\
\hline \multicolumn{6}{|l|}{$R_{d}(\mu \mathrm{mol} / \mathrm{min} / \mathrm{kgFFM})$} \\
\hline Basal & $11.6 \pm 0.3^{\mathrm{AC}}$ & $10.4 \pm 0.4^{A}$ & $14.5 \pm 1.0^{\mathrm{B}}$ & $12.9 \pm 0.7^{\mathrm{BC}}$ & 0.016 \\
\hline $10 \mathrm{mU}$ & $16.5 \pm 1.0^{\mathrm{A}}$ & $13.0 \pm 1.5^{\mathrm{AB}}$ & $10.0 \pm 0.5^{\mathrm{B}}$ & $11.3 \pm 0.3^{B}$ & $<0.001$ \\
\hline$\delta$ & $4.9 \pm 1.1^{\mathrm{A}}$ & $2.6 \pm 4.4^{\mathrm{AC}}$ & $-4.4 \pm 0.8^{B}$ & $-1.6 \pm 0.8^{\mathrm{BC}}$ & $<0.001$ \\
\hline $40 \mathrm{mU}$ & $45.6 \pm 2.3^{\mathrm{A}}$ & $25.7 \pm 2.7^{B}$ & $28.5 \pm 1.9^{\mathrm{B}}$ & $24.3 \pm 2.5^{\mathrm{B}}$ & $<0.001$ \\
\hline$\delta$ & $34.0 \pm 2.3^{\mathrm{A}}$ & $15.3 \pm 2.8^{B}$ & $14.0 \pm 2.1^{B}$ & $11.4 \pm 2.8^{B}$ & $<0.001$ \\
\hline \multicolumn{6}{|l|}{$\begin{array}{l}\text { Glucose oxidation } \\
(\mu \mathrm{mol} / \mathrm{min} / \mathrm{kgFFM})\end{array}$} \\
\hline Basal & $9.0 \pm 2.4$ & $8.1 \pm 2.0$ & $8.4 \pm 1.0$ & $4.9 \pm 1.5$ & 0.466 \\
\hline $10 \mathrm{mU}$ & $13.8 \pm 1.1^{\mathrm{A}}$ & $8.7 \pm 1.8^{\mathrm{B}}$ & $11.5 \pm 0.9^{\mathrm{AB}}$ & $6.9 \pm 0.6^{\mathrm{B}}$ & 0.001 \\
\hline$\delta$ & $4.8 \pm 2.1$ & $0.6 \pm 1.4$ & $3.1 \pm 0.4$ & $2.0 \pm 1.9$ & 0.381 \\
\hline $40 \mathrm{mU}$ & $22.2 \pm 2.3^{\mathrm{A}}$ & $16.7 \pm 2.1^{\mathrm{AB}}$ & $16.4 \pm 0.9^{\mathrm{AB}}$ & $13.5 \pm 0.9^{B}$ & 0.020 \\
\hline$\delta$ & $13.2 \pm 1.1^{\mathrm{A}}$ & $8.6 \pm 1.4^{\mathrm{AB}}$ & $8.0 \pm 1.2^{\mathrm{B}}$ & $8.5 \pm 2.1^{\mathrm{AB}}$ & 0.037 \\
\hline \multicolumn{6}{|l|}{ NOGD $(\mu \mathrm{mol} / \mathrm{min} / \mathrm{kgFFM})$} \\
\hline Basal & $2.8 \pm 2.2$ & $2.0 \pm 1.9$ & $6.1 \pm 1.7$ & $7.9 \pm 1.5$ & 0.134 \\
\hline $10 \mathrm{mU}$ & $3.1 \pm 1.7^{\mathrm{AB}}$ & $5.1 \pm 1.1^{\mathrm{A}}$ & $-1.4 \pm 0.8^{\mathrm{B}}$ & $4.4 \pm 0.7^{\mathrm{A}}$ & 0.004 \\
\hline$\delta$ & $0.4 \pm 3.0^{\mathrm{AB}}$ & $3.1 \pm 2.1^{\mathrm{B}}$ & $-7.5 \pm 0.9^{\mathrm{AB}}$ & $-3.5 \pm 1.8^{\mathrm{AB}}$ & 0.008 \\
\hline $40 \mathrm{mU}$ & $23.6 \pm 2.3^{\mathrm{A}}$ & $11.3 \pm 1.3^{\mathrm{B}}$ & $12.1 \pm 2.5^{\mathrm{B}}$ & $10.8 \pm 2.1^{\mathrm{B}}$ & $<0.001$ \\
\hline$\delta$ & $20.8 \pm 3.2^{\mathrm{A}}$ & $9.3 \pm 2.2^{\mathrm{B}}$ & $6.1 \pm 2.1^{\mathrm{B}}$ & $2.9 \pm 3.1^{\mathrm{B}}$ & $<0.001$ \\
\hline \multicolumn{6}{|l|}{$\begin{array}{l}\text { Fat oxidation } \\
(\mu \mathrm{mol} / \mathrm{min} / \mathrm{kgFFM})\end{array}$} \\
\hline Basal & $5.5 \pm 0.4$ & $5.0 \pm 0.4$ & $5.2 \pm 0.2$ & $5.9 \pm 0.5$ & 0.482 \\
\hline $10 \mathrm{mU}$ & $3.7 \pm 0.3$ & $4.4 \pm 0.5$ & $4.0 \pm 0.1$ & $5.0 \pm 0.5$ & 0.184 \\
\hline$\delta$ & $-1.8 \pm 0.5$ & $-0.6 \pm 0.3$ & $-1.2 \pm 0.2$ & $-0.9 \pm 0.5$ & 0.250 \\
\hline $40 \mathrm{mU}$ & $2.0 \pm 0.4$ & $2.8 \pm 0.5$ & $2.8 \pm 0.3$ & $3.5 \pm 0.6$ & 0.216 \\
\hline$\delta$ & $-3.5 \pm 0.3$ & $-2.2 \pm 0.3$ & $-2.4 \pm 0.2$ & $-2.4 \pm 0.5$ & 0.052 \\
\hline \multicolumn{6}{|l|}{ Plasma FFA ( $\mu \mathrm{mol} / \mathrm{l})$} \\
\hline Basal & $657.6 \pm 46.7$ & $726.1 \pm 54.5$ & $647.9 \pm 45.6$ & $707.3 \pm 24.7$ & 0.574 \\
\hline $10 \mathrm{mU}$ & $193.4 \pm 17.6^{\mathrm{A}}$ & $317.4 \pm 33.2^{\mathrm{B}}$ & $229.3 \pm 18.2^{\mathrm{A}}$ & $322.5 \pm 17.4^{\mathrm{B}}$ & 0.008 \\
\hline$\delta \%$ & $-69.6 \pm 3.3^{\mathrm{A}}$ & $-54.5 \pm 5.0^{\mathrm{B}}$ & $-63.2 \pm 4.7^{\mathrm{AB}}$ & $-54.3 \pm 2.5^{\mathrm{AB}}$ & 0.041 \\
\hline $40 \mathrm{mU}$ & $98.3 \pm 13.5$ & $149.4 \pm 21.3$ & $119.5 \pm 27.9$ & $174.7 \pm 18.3$ & 0.080 \\
\hline$\delta \%$ & $-85.3 \pm 1.4^{\mathrm{A}}$ & $-80.0 \pm 1.7^{\mathrm{AB}}$ & $-81.3 \pm 4.4^{\mathrm{AB}}$ & $-75.4 \pm 5.0^{\mathrm{B}}$ & 0.049 \\
\hline
\end{tabular}

EGP: endogenous glucose production; FFA: free fatty acids; $M$-value: mean glucose infusion rate; $M / I$ ratio: $M$-value per mean plasma insulin concentration (I); NOGD: non-oxidative glucose disposal; $R_{\mathrm{d}}$ : glucose disposal. Different letters indicate significant differences between groups $(P<0.05)$. 
A
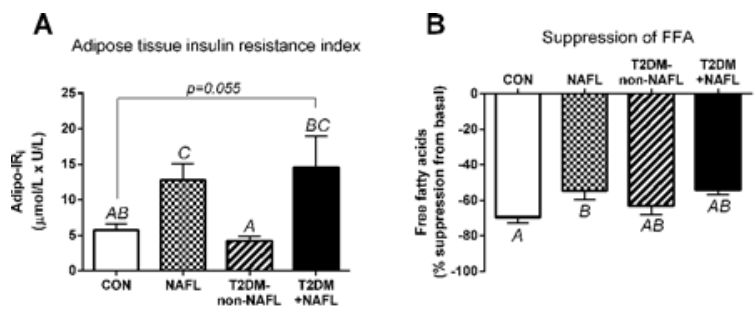

C

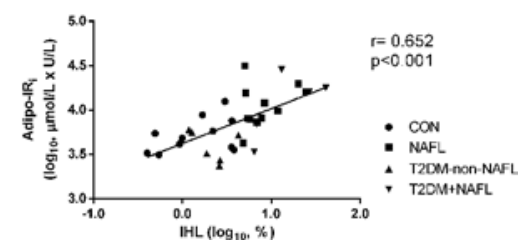

Figure 2. Adipose tissue insulin sensitivity

(A) Fasting adipose tissue insulin resistance index (Adipo-IR $\mathrm{R}_{\mathrm{i}}=$ fasting plasma FFA ( $\left.\mu \mathrm{mol} / \mathrm{I}\right) \times$ fasting plasma insulin concentrations $\left.(\mathrm{U} / \mathrm{L})\right)$; (B) percentage suppression of plasma FFA during $10 \mathrm{mU} / \mathrm{m}^{2} / \mathrm{min}$ insulin infusion; (C) relation between Adipo- $/ \mathrm{R}_{\mathrm{i}}$ and intrahepatic lipid (IHL) content $(n=33)$. Results are mean \pm SEM. Different letters indicate significant differences between groups $(P<0.05)$.
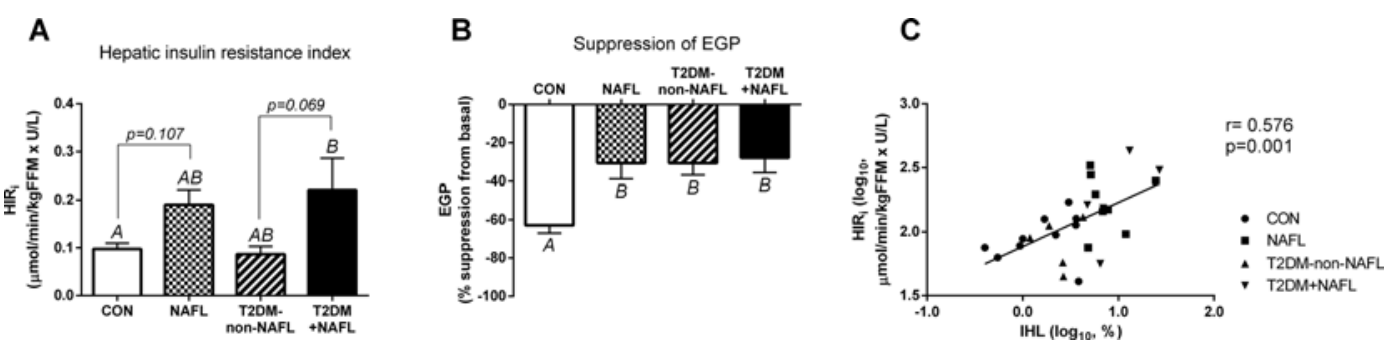

Figure 3. Hepatic insulin sensitivity

(A) Fasting hepatic insulin resistance index $\left(\mathrm{HIR}_{\mathrm{i}}=\right.$ fasting EGP $(\mu \mathrm{mol} / \mathrm{min} / \mathrm{kgFFM}) \times$ fasting plasma insulin concentrations $\left.(\mathrm{U} / \mathrm{l})\right)$; (B) percentage suppression of EGP during $10 \mathrm{mU} / \mathrm{m}^{2} / \mathrm{min}$ insulin infusion; (C) relation between $\mathrm{HIR}_{\mathrm{i}}$ and intrahepatic lipid (IHL) content $(n=28)$. Results are mean \pm SEM. Different letters indicate significant differences between groups $(P<0.05)$.

CON subjects $(P=0.961$, Figure $2 A)$. NAFL subjects were characterized by higher Adipo-IR $R_{\mathrm{i}}$ in comparison with CON subjects $(P=0.003)$ and T2DM-non-NAFL subjects $(P=0.046)$. T2DM+NAFL were also characterized by higher Adipo- $\mathrm{IR}_{\mathrm{i}}$ in comparison with T2DM-non-NAFL $(P=0.042)$ and $\mathrm{CON}(P=0.055)$, with the latter difference being almost significant (Figure 2A). These results suggest that high IHL content, either with or without T2DM, is associated with reduced adipose tissue insulin sensitivity under fasting conditions.

A more dynamic measure of adipose tissue insulin sensitivity is the insulin-stimulated relative suppression of plasma FFA during $10 \mathrm{mU} / \mathrm{m}^{2} / \mathrm{min}$ insulin infusion. Again, suppression of plasma FFA was significantly different among groups $(P=0.041$, Figure $2 \mathrm{~B}$, Table 2$)$. Suppression of plasma FFA was lower in NAFL than in CON subjects $(P=0.046$, Figure $2 \mathrm{~B}$, Table 2$)$, with T2DM-non-NAFL and T2DM+NAFL having intermediate values (Figure $2 \mathrm{~B}$, Table 2), suggesting that subjects with NAFL are characterized by adipose tissue insulin resistance comparable with levels in T2DM, independent of IHL content in T2DM.

Suppression of plasma FFA showed a weak negative correlation with IHL content in the whole study population $(r=-0.355, P=0.046)$, while Adipo-IR $\mathrm{I}_{\mathrm{i}}$ showed a strong positive correlation with IHL content in the whole study population $(r=0.652, P<0.001$, Figure $2 \mathrm{C})$.

\section{Hepatic insulin sensitivity}

Hepatic insulin resistance during basal conditions was determined by using the validated $\mathrm{HIR}_{\mathrm{i}}[12]$. $\mathrm{HIR}_{\mathrm{i}}$ was significantly different among groups $(P=0.016$, Figure $3 \mathrm{~A})$. $\mathrm{HIR}_{\mathrm{i}}$ in NAFL was comparable with $\mathrm{HIR}_{\mathrm{i}}$ in $\mathrm{T} 2 \mathrm{DM}+\mathrm{NAFL}$ $(P=0.908$, Figure $3 \mathrm{~A})$. $\mathrm{HIR}_{\mathrm{i}}$ in $\mathrm{T} 2 \mathrm{DM}$-non-NAFL was comparable with $\mathrm{HIR}_{\mathrm{i}}$ in $\mathrm{CON}(P=0.995$, Figure $3 \mathrm{~A})$. T2DM+NAFL were characterized by higher $\mathrm{HIR}_{\mathrm{i}}$ in comparison with CON $(v=0.050)$ and T2DM-non-NAFL $(P=0.069)$, with the latter difference being almost significant (Figure 3A). While NAFL also tended to have higher HIRi than CON $(P=0.107)$ and T2DM-non-NAFL $(P=0.147)$, this did not reach significance (Figure 3A).

Hepatic insulin sensitivity is best determined by suppression of EGP during $10 \mathrm{mU} / \mathrm{m}^{2} / \mathrm{min}$ insulin infusion. Suppression of EGP was different among groups $(P<0.001$, Figure 3B, Table 2). Suppression of EGP was comparable between NAFL and T2DM-non-NAFL subjects $(P=0.999)$; between NAFL and T2DM+NAFL subjects $(P=0.993)$; and between T2DM-non-NAFL and T2DM+NAFL subjects $(P=0.994)$ (Figure 3B, Table 2$)$. NAFL $(P=0.003)$, T2DM-non-NAFL $(P=0.007)$ and T2DM+NAFL subjects $(P=0.006)$ were all characterized by a lower ability to suppress EGP compared with CON subjects (Figure 3B, Table 2), suggesting that subjects with NAFL are characterized by 

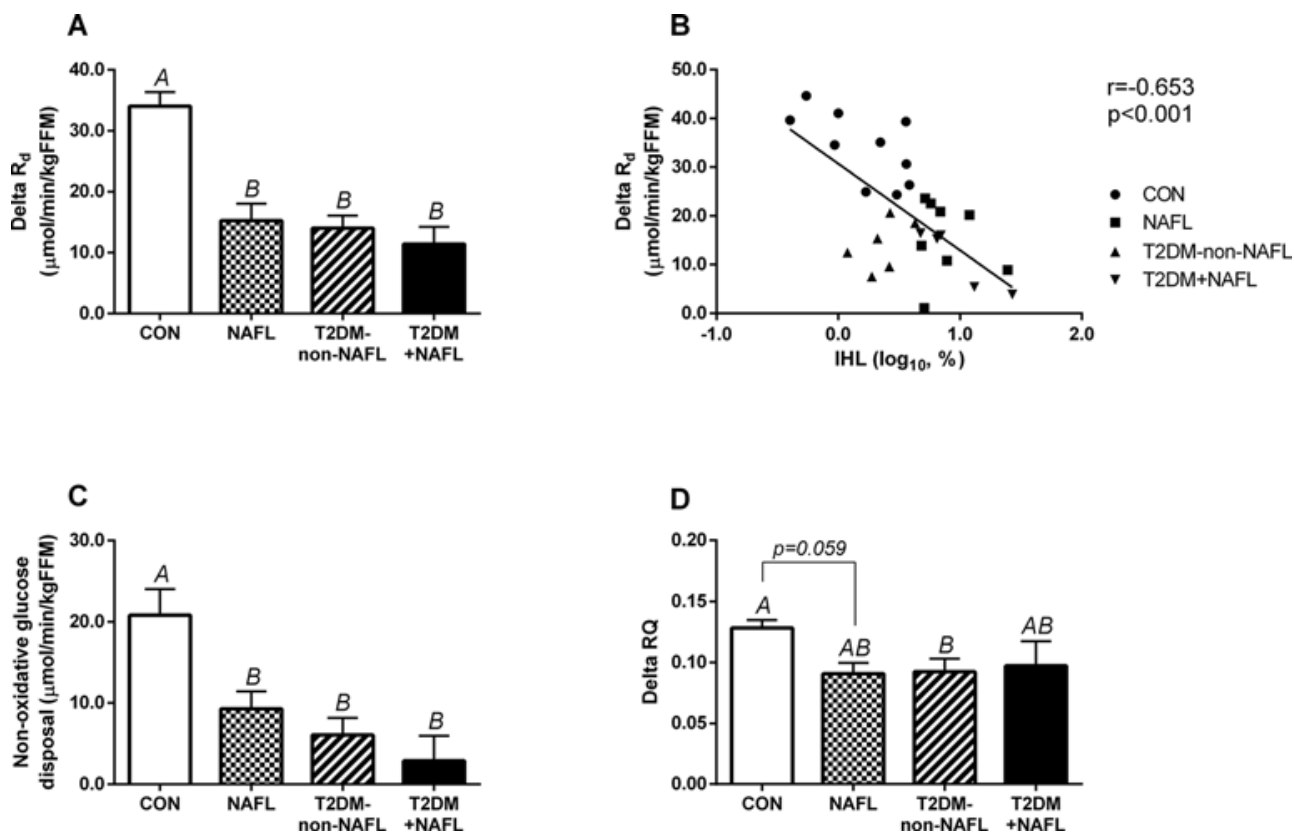

Figure 4. Peripheral insulin sensitivity

(A) Insulin-stimulated glucose disposal $\left(\Delta R_{\mathrm{d}}\right)$ in skeletal muscle during $40 \mathrm{mU} / \mathrm{m}^{2} / \mathrm{min}$ insulin infusion; (B) relation between $\Delta R_{\mathrm{d}}$ and intrahepatic lipid (IHL) content ( $n=29$ ); (C) insulin-stimulated NOGD in skeletal muscle during $40 \mathrm{mU} / \mathrm{m}^{2} / \mathrm{min}$ insulin infusion; (D) metabolic flexibility $(\triangle R Q)$. Results are mean \pm SEM. Different letters indicate significant differences between groups $(P<0.05)$.

hepatic insulin resistance comparable with levels observed in T2DM patients, independent of IHL content in T2DM patients.

Suppression of EGP did not significantly correlate with IHL content in the whole study population $(r=-0.344$, $P=0.068)$. $\mathrm{HIR}_{\mathrm{i}}$, however, did show a positive correlation with IHL content in the whole study population $(P=0.567$, $r=0.001$, Figure 3C).

\section{Peripheral insulin sensitivity}

Peripheral insulin sensitivity, dominated by skeletal muscle insulin sensitivity, was measured as insulin-stimulated glucose uptake $\left(\Delta R_{\mathrm{d}}\right)$, the difference between $R_{\mathrm{d}}$ under insulin-stimulated conditions $\left(40 \mathrm{mU} / \mathrm{m}^{2} / \mathrm{min}\right)$ and $\mathrm{R}_{\mathrm{d}}$ under basal conditions. $\Delta R_{\mathrm{d}}$ was different among groups $(P<0.001) . \Delta R_{\mathrm{d}}$ was comparable between NAFL and T2DM-non-NAFL subjects $(P=0.988)$; between NAFL and T2DM+NAFL subjects $(P=0.768)$; and between T2DM-non-NAFL and T2DM+NAFL subjects $(P=0.925)$ (Figure 4A, Table 2). NAFL $(P<0.001)$, T2DM-non-NAFL $(P<0.001)$ and T2DM+NAFL subjects $(P<0.001)$ were all characterized by a significantly lower $\Delta R_{\mathrm{d}}$ compared with CON subjects (Figure $4 \mathrm{~A}$, Table 2). $\Delta R_{\mathrm{d}}$ correlated negatively with IHL content in the whole study population $(r=-0.653, P<0.001$, Figure $4 \mathrm{~B})$, suggesting that subjects with NAFL are characterized by peripheral insulin resistance comparable with levels observed in T2DM patients.

NOGD was different among groups $(P<0.001$, Figure 4C, Table 2$)$. NOGD was comparable between NAFL and T2DM-non-NAFL subjects $(P=0.820)$; between NAFL and T2DM+NAFL subjects $(P=0.376)$; and between T2DM-non-NAFL and T2DM+NAFL subjects $(P=0.844)$ (Figure $4 \mathrm{C}$, Table 2$)$. NAFL $(P=0.026)$, T2DM-non-NAFL $(P=0.004)$ and T2DM+NAFL subjects $(P=0.001)$ were all characterized by a lower NOGD compared with CON subjects (Figure 4C, Table 2).

\section{Metabolic flexibility}

Metabolic flexibility $(\Delta \mathrm{RQ})$, the difference in RQ under insulin-stimulated conditions $\left(40 \mathrm{mU} / \mathrm{m}^{2} / \mathrm{min}\right)$ and $\mathrm{RQ}$ under basal conditions, was different among groups $(P=0.028$, Figure $4 \mathrm{D}) . \triangle \mathrm{RQ}$ was comparable between NAFL and T2DM-non-NAFL subjects $(P=0.948)$, between NAFL and T2DM+NAFL subjects $(P=0.969)$ and between T2DM-non-NAFL and T2DM+NAFL subjects $(P=0.861$ ) (Figure 4D). NAFL, T2DM-non-NAFL and T2DM+NAFL subjects showed lower metabolic flexibility compared with CON (Figure 4D). The difference was significant between CON and T2DM-non-NAFL subjects $(P=0.047)$, while the difference between CON and NAFL subjects $(P=0.059)$ 
was almost significant (Figure 4D). The difference between CON and T2DM+NAFL subjects was not significant $(P=0.457$, Figure 4D).

\section{Discussion}

In the current study, we show that non-diabetic individuals with NAFL are metabolically impaired to a similar degree as BMI-matched patients with T2DM. Moreover, our data suggests that elevated IHL content seems to be a contributing determinant of adipose tissue insulin resistance under fasting conditions, hepatic insulin resistance under fasting conditions and insulin-stimulated peripheral insulin resistance in individuals with and without T2DM.

Previous studies revealed that adipose tissue, hepatic and skeletal muscle insulin sensitivity all were impaired in individuals with NAFL in comparison with BMI-matched healthy individuals with normal IHL content [15-18]. Thus, we anticipated that adipose tissue, hepatic and skeletal muscle insulin sensitivity and metabolic flexibility in NAFL subjects would have intermediate values when compared with insulin-resistant T2DM patients and more insulin-sensitive CON subjects. In contrast with this expectation, here we demonstrated that adipose tissue, hepatic and skeletal muscle insulin sensitivity and metabolic flexibility were all impaired in subjects with NAFL to a similar degree to that observed in T2DM patients-irrespective of IHL content-who were marked by hyperglycemia at onset of the study (FPG $\geqslant 7.0 \mathrm{mmol} / \mathrm{l}$ and $<10.0 \mathrm{mmol} / \mathrm{l}$ ) while on glucose lowering medication.

When the whole study population was taken together, IHL content showed strong associations with markers of adipose tissue, and hepatic insulin resistance measured under fasting conditions, and with skeletal muscle insulin resistance measured using a hyperinsulinemic-euglycemic clamp. Considering NAFL and CON subjects together, we found an association between IHL content and plasma glucose levels upon OGTT. A recent study investigating surrogate indexes of insulin resistance in NAFL patients found that-independent of obesity-plasma glucose levels upon OGTT were different when NAFL patients were divided into groups of low and high liver fibrosis, while plasma glucose levels were not different when NAFL patients were divided into groups of low and high steatosis [34]. We did not measure the state of fibrosis in this study. Therefore, it cannot be ruled out that some of our NAFL subjects were characterized by having significant fibrosis of the liver, which might have been responsible for the lower glucose tolerance in these subjects compared with CON subjects.

Despite higher IHL content in NAFL and in T2DM+NAFL subjects compared with CON and T2DM-non-NAFL subjects, the transaminases AST and ALT-two serum markers for liver disease-were not significantly different between these groups. Even though AST and ALT concentrations in most participants were within the suggested normal clinical range (AST: 4-40U/L; ALT: 1-45U/L) [35], we did observe a positive correlation between AST, and ALT with IHL content. This observation agrees with earlier studies showing that liver enzymes do associate with IHL content [35-38]. Therefore, our results are in line with previous reports showing that significant increases in IHL content measured with ${ }^{1} \mathrm{H}-\mathrm{MRS}$ reflected only modest elevations in plasma transaminase levels $[37,39,40]$, and that direct measurement of IHL content is warranted to detect early stages of NAFL [41].

The average elevation in IHL content was not significantly different between NAFL subjects and T2DM+NAFL patients; and CON subjects and T2DM-non-NAFL patients also had similarly low levels of IHL content. This is of importance when comparing these groups considering that the IHL content is directly correlated with markers for insulin resistance, as shown in the present study and previous studies [9-14]. Of note, 11 T2DM patients were treated with metformin, a glucose-lowering drug known to decrease IHL content [42]. Metformin use was comparable between T2DM patients with normal IHL content $(n=5)$ and T2DM patients with high IHL content $(n=6)$, but might have resulted in slight underestimations of the deteriorations in patients with T2DM.

In this study, individuals with NAFL had profound resistance to the insulin-stimulated suppression of plasma FFA in comparison with healthy individuals with normal IHL content, with intermediate values observed in patients with T2DM. Previous research described that patients with NAFL had lower insulin-stimulated suppression of plasma FFA than healthy individuals with normal IHL content $[6,15,35,43,44]$. Other studies have reported impaired suppression of plasma FFA during the OGTT measurement in patients with NASH [45] and NAFL [40] in comparison with individuals without fatty liver disease, in the absence [45] and presence [40,45] of T2DM. No previous study, however, compared insulin-stimulated suppression of plasma FFA in patients with NAFL to T2DM patients. Our observation was unexpected, and suggests that the insulin resistance in adipose tissue is part of an impaired substrate handling in individuals with NAFL, who are otherwise healthy.

In addition to the above, we found higher Adipo- $\mathrm{IR}_{\mathrm{i}}-\mathrm{a}$ validated predictor of adipose tissue insulin sensitivity under fasting conditions [33] - in NAFL subjects and T2DM+NAFL patients in comparison with CON subjects and T2DM-non-NAFL patients, as well as a strong association of Adipo- $\mathrm{IR}_{\mathrm{i}}$ with IHL content. A recent study in 302 subjects with different degrees of glucose tolerance concluded that Adipo- $\mathrm{IR}_{\mathrm{i}}$ increased in the transition from normal 
glucose tolerance to $\mathrm{T} 2 \mathrm{DM}$, with Adipo- $\mathrm{IR}_{\mathrm{i}}$ increasing from subjects with normal glucose tolerance, over subjects with impaired glucose tolerance, to patients with T2DM [46]. Our results are somewhat different from those recent results, given that our T2DM-non-NAFL patients were not characterized by higher Adipo-IR $\mathrm{R}_{\mathrm{i}}$ compared with CON subjects, while our NAFL subjects had high Adipo-IR $\mathrm{R}_{\mathrm{i}}$, comparable with our T2DM+NAFL patients. It is known, however, that the prevalence of NAFL also increases from subjects with normal glucose tolerance, over subjects with impaired glucose tolerance, to patients with T2DM [6,47]. Thus, differences in the prevalence of NAFL in the subpopulations studied in [46] might have been a confounding factor contributing to the higher Adipo- $\mathrm{IR}_{\mathrm{i}}$.

We indeed found a strong association between Adipo-IR $\mathrm{I}_{\mathrm{i}}$ and IHL content. While basal plasma FFA were somewhat higher in NAFL and T2DM+NAFL subjects, the higher Adipo- $\mathrm{IR}_{\mathrm{i}}$ mainly originated from higher basal insulin levels in NAFL and T2DM+NAFL subjects compared with CON and T2DM-non-NAFL subjects. Thus, all subjects with NAFL were characterized by a higher resistance to the antilipolytic effect of insulin [46]. Therefore, our data seems to indicate that Adipo- $\mathrm{IR}_{\mathrm{i}}$ is more closely associated with the development of NAFL than with the development of T2DM perse.

Elevated IHL content has been shown to be closely related to hepatic insulin resistance [12]. Here, we show that high IHL content impairs the suppression of EGP to a similar degree as in patients with T2DM, but that, in T2DM patients, suppression of EGP is impaired independently of IHL content. Of note, visceral adipose tissue mass is as strongly related to suppression of EGP as IHL content [47], and correlates closely with IHL content itself [12,47]. We did not measure visceral adipose tissue mass in this study, but higher visceral adipose tissue mass might have contributed to the impaired suppression of EGP as well.

While elevated IHL content is related to hepatic insulin resistance, Ortiz-Lopez et al. [6] previously observed that suppression of EGP in individuals with NAFL and normal glucose tolerance was not different from individuals without NAFL. However, this observation was only true in individuals with normal glucose tolerance, since individuals with NAFL who suffered from pre-diabetes or T2DM did have lower suppression of EGP [6,35]. Our data support the latter, since our NAFL subjects also had elevated plasma glucose values upon OGTT. Moreover, our T2DM-non-NAFL patients were also characterized by lower suppression of EGP. Thus, impaired glucose handling rather than elevated IHL content seems to be related to impaired suppression of EGP.

The $\mathrm{HIR}_{\mathrm{i}}$ has been described to be lower in subjects with NAFL in comparison with healthy individuals $[6,15,16,35]$. In the present study, $\mathrm{HIR}_{\mathrm{i}}$ correlated with IHL content as previously observed in non-diabetic obese subjects $[9,48]$. Furthermore, in contrast with suppression of EGP, T2DM+NAFL patients had higher HIR $\mathrm{H}_{\mathrm{i}}$ values than T2DM-non-NAFL patients, with $\mathrm{HIR}_{\mathrm{i}}$ values being comparable between T2DM non-NAFL patients and CON individuals. Therefore, one could suggest that hepatic insulin sensitivity under fasting conditions is more dependant on IHL content than the insulin-stimulated suppression of EGP.

The present study demonstrated that NAFL subjects were characterized by similar skeletal muscle insulin resistance than patients with T2DM, and confirmed previous results showing lower rates of insulin-stimulated glucose disposal in individuals with NAFL than in healthy subjects with normal IHL content $[6,15,16,44]$. Insulin resistance in skeletal muscle is featured by lower metabolic flexibility, a highly important determinant of T2DM development [49], and known to be impaired in subjects with NAFL compared with CON individuals [44]. Therefore, we measured metabolic flexibility during the hyperinsulinemic-euglycemic clamp. We found a similar impairment in metabolic flexibility in NAFL subjects as in T2DM-non-NAFL and T2DM+NAFL patients, which was reflected by a lower increase in RQ upon high insulin infusion in comparison with CON subjects. In other groups of subjects with prediabetes, metabolic flexibility and skeletal muscle insulin resistance have been shown to be lower than in BMI-matched healthy subjects, although values were still higher than those observed in T2DM patients [19,20]. Thus, our data suggest that individuals with NAFL have more severe insulin resistance of the skeletal muscle than other groups at risk of developing T2DM, and in fact have insulin resistance to a level similar to that seen in T2DM patients.

An association between skeletal muscle insulin resistance and IHL content has been previously described in healthy obese subjects [9-11,50] and individuals with NAFL [50]. Here, we show that this association still persists when NAFL, T2DM-non-NAFL, T2DM+NAFL, and CON individuals are merged and studied together. However, skeletal muscle insulin resistance was not different between T2DM-non-NAFL and T2DM+NAFL patients, and T2DM-non-NAFL patients were more insulin resistant at the level of the skeletal muscle than CON subjects. Thus, although across the four groups, higher amounts of IHL content did associate with lower levels of insulin sensitivity in skeletal muscle, insulin resistance in the skeletal muscle of patients with T2DM did not develop solely in the presence of NAFL, unmasking other causes (of yet-to-be-determined origin) related to the development of insulin resistance of the skeletal muscle in patients with T2DM. 
In conclusion, we show that non-diabetic individuals with NAFL suffer from metabolic perturbations to a similar degree as patients with T2DM. These observations suggest that NAFL is an important feature leading to severe insulin resistance and should be viewed as a serious health threat for the development of T2DM.

\section{Clinical Perspectives}

- Non-alcoholic fatty liver (NAFL) is the most common liver disorder in western society and is closely related to the development of insulin resistance and type 2 diabetes (T2DM). However, the degree to which metabolic complications typical for T2DM already develop in individuals with NAFL is unknown.

- Adipose tissue, hepatic and skeletal muscle insulin sensitivity, and metabolic flexibility were all severely impaired in individuals with NAFL in comparison with those with normal intrahepatic lipid, and these impairments were comparable with those observed in patients with T2DM.

- NAFL is an important feature leading to severe insulin resistance and should be viewed as a serious health threat for the development of T2DM.

\section{Acknowledgments}

We greatly acknowledge all research volunteers for participating in the study. We thank P. Schoffelen (Maastricht University Medical Center) for the technical support.

\section{Competing Interests}

The authors declare that there are no competing interests associated with the manuscript.

\section{Funding}

The project is supported by the Dutch Diabetes Research Foundation [grant number 2009.60.003]; Helmholtz Alliance with Universities (Imaging and Curing Environmental Metabolic Diseases, ICEMED) (to T.J. and M.R.); German Research Foundation [grant number DFG, SFB 1116, B05 (to T.J and M.R.)]; and Schmutzler-Stiftung (to T.J. and M.R.).

\section{Author Contribution}

B.B. designed the study, performed experiments, analyzed data, and wrote the manuscript; V.B.S. designed the study, analyzed data, critically reviewed, and edited the manuscript; T.J. critically reviewed and edited the manuscript; A.G. performed experiments and analyzed data; B.H. critically reviewed and edited the manuscript; Y.B. performed experiments; D.D. performed experiments; M.R. critically reviewed and edited the manuscript; M.K.C.H. designed the study, analyzed data, and critically reviewed and edited the manuscript; P.S. designed the study, analyzed data, and critically reviewed and edited the manuscript. P.S. is the guarantor of this work and, as such, had full access to all the data in the study and takes responsibility for the integrity of the data and the accuracy of the data analysis.

\section{Abbreviations}

ALAT, alanine aminotransferase; ASAT, aspartate aminotransferase; ATP, adenosine triphosphate; BMI, body mass index; CON, control; EGP, endogenous glucose production; FFA, free fatty acids; FPG, fasting plasma glucose; GGT, g-glutamyl transpeptidase; HDL, high-density lipoprotein; ${ }^{1} \mathrm{HMRS}$, Proton magnetic resonance spectroscopy; IHL, intrahepatic lipid; LDL, low-density lipoprotein; NAFL, non-alcoholic fatty liver; NOGD, non-oxidative glucose disposal; $R_{a}$, rate of appearance; $R_{d}$, rate of disappearance; T2DM, type 2 diabetes mellitus; TG, triglycerides; $\mathrm{VO}_{2 \max }$, maximal oxidative capacity.

\section{References}

1 Caldwell, S. and Argo, C. (2010) The natural history of non-alcoholic fatty liver disease. Dig. Dis. 28, 162-168

2 Tilg, H., Moschen, A.R. and Roden, M. (2017) NAFLD and diabetes mellitus. Nature Reviews. Gastroenterol. Hepatol. 14, 32-42

3 Firneisz, G. (2014) Non-alcoholic fatty liver disease and type 2 diabetes mellitus: the liver disease of our age? World J. Gastroenterol. 20, 9072-9089

4 Utzschneider, K.M. and Kahn, S.E. (2006) Review: The role of insulin resistance in nonalcoholic fatty liver disease. J. Clin. Endocrinol. Metab. 91, 4753-4761

5 Yki-Jarvinen, H. (2010) Liver fat in the pathogenesis of insulin resistance and type 2 diabetes. Dig. Dis. 28, 203-209 
6 Ortiz-Lopez, C., Lomonaco, R., Orsak, B., Finch, J., Chang, Z., Kochunov, V.G. et al. (2012) Prevalence of prediabetes and diabetes and metabolic profile of patients with nonalcoholic fatty liver disease (NAFLD). Diabetes Care 35, 873-878

7 Suh, S.Y., Choi, S.E., Ahn, H.Y., Yang, H.M., Kim, Y.I. and Sung, N.J. (2009) The association between normal alanine aminotransferase levels and the metabolic syndrome: 2005 Korean National Health and Nutrition Examination Survey. Metabolism 58, 1731-1736

8 Ioannou, G.N., Weiss, N.S., Boyko, E.J., Kahn, S.E. and Lee, S.P. (2005) Contribution of metabolic factors to alanine aminotransferase activity in persons with other causes of liver disease. Gastroenterology 128, 627-635

9 Korenblat, K.M., Fabbrini, E., Mohammed, B.S. and Klein, S. (2008) Liver, muscle, and adipose tissue insulin action is directly related to intrahepatic triglyceride content in obese subjects. Gastroenterology 134, 1369-1375

10 Koska, J., Stefan, N., Permana, P.A., Weyer, C., Sonoda, M., Bogardus, C. et al. (2008) Increased fat accumulation in liver may link insulin resistance with subcutaneous abdominal adipocyte enlargement, visceral adiposity, and hypoadiponectinemia in obese individuals. Am. J. Clin. Nutr. 87, 295-302

11 Hwang, J.H., Stein, D.T., Barzilai, N., Cui, M.H., Tonelli, J., Kishore, P. et al. (2007) Increased intrahepatic triglyceride is associated with peripheral insulin resistance: in vivo MR imaging and spectroscopy studies. Am. J. Physiol. Endocrinol. Metab. 293, E1663-1669

12 Gastaldelli, A., Cusi, K., Pettiti, M., Hardies, J., Miyazaki, Y., Berria, R. et al. (2007) Relationship between hepatic/visceral fat and hepatic insulin resistance in nondiabetic and type 2 diabetic subjects. Gastroenterology 133, 496-506

13 Roden, M. (2006) Mechanisms of Disease: hepatic steatosis in type 2 diabetes-pathogenesis and clinical relevance. Nature clinical practice. Endocrinol. Metab. 2, 335-348

14 Kato, K., Takamura, T., Takeshita, Y., Ryu, Y., Misu, H., Ota, T. et al. (2014) Ectopic fat accumulation and distant organ-specific insulin resistance in Japanese people with nonalcoholic fatty liver disease. PLOS ONE 9, e92170

15 Fabbrini, E., Magkos, F., Mohammed, B.S., Pietka, T., Abumrad, N.A., Patterson, B.W. et al. (2009) Intrahepatic fat, not visceral fat, is linked with metabolic complications of obesity. Proc. Natl. Acad. Sci. U.S.A. 106, 15430-15435

16 Deivanayagam, S., Mohammed, B.S., Vitola, B.E., Naguib, G.H., Keshen, T.H., Kirk, E.P. et al. (2008) Nonalcoholic fatty liver disease is associated with hepatic and skeletal muscle insulin resistance in overweight adolescents. Am. J. Clin. Nutr. 88, 257-262

17 Lomonaco, R., Ortiz-Lopez, C., Orsak, B., Webb, A., Hardies, J., Darland, C. et al. (2012) Effect of adipose tissue insulin resistance on metabolic parameters and liver histology in obese patients with nonalcoholic fatty liver disease. Hepatology 55, 1389-1397

18 Szendroedi, J., Zwettler, E., Schmid, A.I., Chmelik, M., Pacini, G., Kacerovsky, G. et al. (2008) Reduced basal ATP synthetic flux of skeletal muscle in patients with previous acromegaly. PLOS ONE 3, e3958

19 Phielix, E., Schrauwen-Hinderling, V.B., Mensink, M., Lenaers, E., Meex, R., Hoeks, J. et al. (2008) Lower intrinsic ADP-stimulated mitochondrial respiration underlies in vivo mitochondrial dysfunction in muscle of male type 2 diabetic patients. Diabetes 57, 2943-2949

20 Mohd Nor, N.S., Lee, S., Bacha, F., Tfayli, H. and Arslanian, S. (2016) Triglyceride glucose index as a surrogate measure of insulin sensitivity in obese adolescents with normoglycemia, prediabetes, and type 2 diabetes mellitus: comparison with the hyperinsulinemic-euglycemic clamp. Pediatr. Diab. 17, 458-465

21 Kuipers, H., Verstappen, F.T., Keizer, H.A., Geurten, P. and van Kranenburg, G. (1985) Variability of aerobic performance in the laboratory and its physiologic correlates. Int. J. Sports Med. 6, 197-201

22 Bilet, L., Brouwers, B., van Ewijk, P.A., Hesselink, M.K., Kooi, M.E., Schrauwen, P. et al. (2015) Acute exercise does not decrease liver fat in men with overweight or NAFLD. Sci. Rep. 5, 9709

23 Lindeboom, L., Nabuurs, C.I., Hesselink, M.K., Wildberger, J.E., Schrauwen, P. and Schrauwen-Hinderling, V.B. (2015) Proton magnetic resonance spectroscopy reveals increased hepatic lipid content after a single high-fat meal with no additional modulation by added protein. Am. J. Clin. Nutr. 101, $65-71$

24 Guiu, B., Petit, J.M., Loffroy, R., Ben Salem, D., Aho, S., Masson, D. et al. (2009) Quantification of liver fat content: comparison of triple-echo chemical shift gradient-echo imaging and in vivo proton MR spectroscopy. Radiology 250, 95-102

25 Szczepaniak, L.S., Nurenberg, P., Leonard, D., Browning, J.D., Reingold, J.S., Grundy, S. et al. (2005) Magnetic resonance spectroscopy to measure hepatic triglyceride content: prevalence of hepatic steatosis in the general population. Am. J. Physiol. Endocrinol. Metab. 288, E462-E468

26 DeFronzo, R.A., Tobin, J.D. and Andres, R. (1979) Glucose clamp technique: a method for quantifying insulin secretion and resistance. Am. J. Physiol. 237, E214-E223

27 van de Weijer, T., Phielix, E., Bilet, L., Williams, E.G., Ropelle, E.R., Bierwagen, A. et al. (2015) Evidence for a direct effect of the NAD+ precursor acipimox on muscle mitochondrial function in humans. Diabetes 64, 1193-1201

28 Finegood, D.T., Bergman, R.N. and Vranic, M. (1987) Estimation of endogenous glucose production during hyperinsulinemic-euglycemic glucose clamps. Comparison of unlabeled and labeled exogenous glucose infusates. Diabetes 36, 914-924

29 Ackermans, M.T., Pereira Arias, A.M., Bisschop, P.H., Endert, E., Sauerwein, H.P. and Romijn, J.A. (2001) The quantification of gluconeogenesis in healthy men by (2) $\mathrm{H} 2 \mathrm{O}$ and [2-(13)C]glycerol yields different results: rates of gluconeogenesis in healthy men measured with (2)H2O are higher than those measured with [2-(13)C]glycerol. J. Clin. Endocrinol. Metab. 86, 2220-2226

30 Steele, R. (1959) Influences of glucose loading and of injected insulin on hepatic glucose output. Ann. N.Y. Acad. Sci. 82, 420-430

31 Peronnet, F. and Massicotte, D. (1991) Table of nonprotein respiratory quotient: an update. Can. J. Sport Sci. 16, 23-29

32 Friedewald, W.T., Levy, R.I. and Fredrickson, D.S. (1972) Estimation of the concentration of low-density lipoprotein cholesterol in plasma, without use of the preparative ultracentrifuge. Clin. Chem. 18, 499-502

33 Sondergaard, E., Espinosa De Ycaza, A.E., Morgan-Bathke, M. and Jensen, M.D. (2017) How to Measure Adipose Tissue Insulin Sensitivity. J. Clin. Endocrinol. Metab. 102, 1193-1199

34 Rosso, C., Mezzabotta, L., Gaggini, M., Salomone, F., Gambino, R., Marengo, A. et al. (2016) Peripheral insulin resistance predicts liver damage in nondiabetic subjects with nonalcoholic fatty liver disease. Hepatology 63, 107-116 
35 Maximos, M., Bril, F., Portillo Sanchez, P., Lomonaco, R., Orsak, B., Biernacki, D. et al. (2015) The role of liver fat and insulin resistance as determinants of plasma aminotransferase elevation in nonalcoholic fatty liver disease. Hepatology 61, 153-160

36 van den Berg, E.H., Amini, M., Schreuder, T.C., Dullaart, R.P., Faber, K.N., Alizadeh, B.Z. et al. (2017) Prevalence and determinants of non-alcoholic fatty liver disease in lifelines: a large Dutch population cohort. PLOS ONE 12, e0171502

37 Sattar, N., Forrest, E. and Preiss, D. (2014) Non-alcoholic fatty liver disease. BMJ 349, g4596

38 Wroblewski, F. (1958) The clinical significance of alterations in transaminase activities of serum and other body fluids. Adv. Clin. Chem. 1, 313-351

39 Browning, J.D., Szczepaniak, L.S., Dobbins, R., Nuremberg, P., Horton, J.D., Cohen, J.C. et al. (2004) Prevalence of hepatic steatosis in an urban population in the United States: impact of ethnicity. Hepatology 40, 1387-1395

40 Portillo-Sanchez, P., Bril, F., Maximos, M., Lomonaco, R., Biernacki, D., Orsak, B. et al. (2015) High prevalence of nonalcoholic fatty liver disease in patients with type 2 diabetes mellitus and normal plasma aminotransferase levels. J. Clin. Endocrinol. Metab. 100, 2231-2238

41 Koplay, M., Sivri, M., Erdogan, H. and Nayman, A. (2015) Importance of imaging and recent developments in diagnosis of nonalcoholic fatty liver disease. World J. Hepatol. 7, 769-776

42 Tamura, Y., Watada, H., Sato, F., Kumashiro, N., Sakurai, Y., Hirose, T. et al. (2008) Effects of metformin on peripheral insulin sensitivity and intracellular lipid contents in muscle and liver of overweight Japanese subjects. Diabetes Obes. Metab. 10, 733-738

43 Seppala-Lindroos, A., Vehkavaara, S., Hakkinen, A.M., Goto, T., Westerbacka, J., Sovijarvi, A. et al. (2002) Fat accumulation in the liver is associated with defects in insulin suppression of glucose production and serum free fatty acids independent of obesity in normal men. J. Clin. Endocrinol. Metab. 87, 3023-3028

44 Bugianesi, E., Gastaldelli, A., Vanni, E., Gambino, R., Cassader, M., Baldi, S. et al. (2005) Insulin resistance in non-diabetic patients with non-alcoholic fatty liver disease: sites and mechanisms. Diabetologia 48, 634-642

45 Gastaldelli, A., Harrison, S.A., Belfort-Aguilar, R., Hardies, L.J., Balas, B., Schenker, S. et al. (2009) Importance of changes in adipose tissue insulin resistance to histological response during thiazolidinedione treatment of patients with nonalcoholic steatohepatitis. Hepatology 50, 1087-1093

46 Gastaldelli, A., Gaggini, M. and DeFronzo, R.A. (2017) Role of adipose tissue insulin resistance in the natural history of type 2 diabetes: results from the San Antonio Metabolism Study. Diabetes 66, 815-822

47 Gaggini, M., Morelli, M., Buzzigoli, E., DeFronzo, R.A., Bugianesi, E. and Gastaldelli, A. (2013) Non-alcoholic fatty liver disease (NAFLD) and its connection with insulin resistance, dyslipidemia, atherosclerosis and coronary heart disease. Nutrients 5, 1544-1560

48 Kotronen, A., Juurinen, L., Tiikkainen, M., Vehkavaara, S. and Yki-Jarvinen, H. (2008) Increased liver fat, impaired insulin clearance, and hepatic and adipose tissue insulin resistance in type 2 diabetes. Gastroenterology 135, 122-130

49 Kelley, D.E. and Mandarino, L.J. (2000) Fuel selection in human skeletal muscle in insulin resistance: a reexamination. Diabetes 49, 677-683

50 Bril, F., Barb, D., Portillo-Sanchez, P., Biernacki, D., Lomonaco, R., Suman, A. et al. (2017) Metabolic and histological implications of intrahepatic triglyceride content in nonalcoholic fatty liver disease. Hepatology 65, 1132-1144 